\title{
Psycho-Physiological Responses to a 4-Month High-Intensity Interval Training-Centered Multidisciplinary Weight-Loss Intervention in Adolescents with Obesity
}

\author{
Marwa Khammassi ${ }^{1,2,3}{ }^{*}$, Maud Miguet ${ }^{1}$, Valérie julian ${ }^{4}$, Charlotte Cardenoux ${ }^{5}$, Yves Boirie ${ }^{6,7,8,9}{ }^{\text {, Martine Duclos }}{ }^{6,7,8,9}$, \\ Bruno Pereira ${ }^{10}$, David Thivel ${ }^{1,7}$ \\ ${ }^{1}$ Clermont Auvergne University, EA 3533, Laboratory of the Metabolic Adaptations to Exercise under Physiological and Pathological Conditions (AME2P), \\ Clermont-Ferrand, France; ${ }^{2}$ Research Unit, Sportive Performance and Physical Rehabilitation, High Institute of Sports and Physical Education of Kef, University of \\ Jendouba, Kef; ${ }^{3}$ Faculty of Science of Bizerte, University of Carthage, Zarzouna, Tunisia; ${ }^{4} \mathrm{CHU}$ med du sport CHU Clermont-Ferrand, Clermont-Ferrand; ${ }^{5} \mathrm{CMI}$ \\ Romagnat, Clermont-Ferrand; ${ }^{6}$ INRA/UMR 1019, Clermont-Ferrand; ${ }^{7}$ CRNH-Auvergne, Clermont-Ferrand; ${ }^{8}$ University Clermont 1, UFR Medicine, Clermont-Ferrand; \\ ${ }^{9}$ Departments of Human Nutrition and Sport Medicine and Functional Explorations, Clermont-Ferrand University Hospital, G. Montpied Hospital, Clermont-Ferrand; \\ ${ }^{10} \mathrm{Clermont-Ferrand}$ University Hospital, Biostatistics Unit (DRCI), Clermont-Ferrand, France
}

Background: To evaluate the impact of 4 months of a high-intensity interval training (HIIT)-centered weightmanagement intervention on health-related quality of life (HR-QOL), health perception (HP) and physical selfperception (PSP) in adolescents with obesity.

Methods: Fifty-six adolescents with obesity (28 girls; mean body mass index [BMl], $35 \pm 4.89 \mathrm{~kg} / \mathrm{m}^{2} ; \mathrm{z}-\mathrm{BMI}$, $2.3 \pm 0.3 ; 11-17$ years) followed a multidisciplinary weight-management intervention composed of nutritional counseling, HIIT program, and health-related therapeutic education. Anthropometric parameters, body composition (dual X-ray absorptiometry), and maximal aerobic capacities (maximal oxygen consumption $\left[\mathrm{VO}_{2 \text { peak }}\right.$ ) were assessed, and self-reported questionnaires were used to assess HR-QOL (36-item short form survey), HP and PSP at baseline (T0) and post-intervention (T1).

Results: Body weight $(92.6 \pm 18.9$ to $85.9 \pm 16.2 \mathrm{~kg}), \mathrm{BMl}\left(35.0 \pm 4.8\right.$ to $\left.32.1 \pm 4.5 \mathrm{~kg} / \mathrm{m}^{2}\right), \mathrm{z}-\mathrm{BMl}(2.3 \pm 0.3$ to $2.1 \pm 0.3)$ and fat mass percentage $(36.0 \% \pm 9.1 \%$ to $30.4 \% \pm 7.8 \%)$ were significantly decreased $(P<0.001)$ between $\mathrm{T} 0$ and T1. There was a tendency for $\mathrm{VO}_{2 \text { peak }}$ to increase from $26.35 \pm 5.81 \mathrm{~mL} / \mathrm{kg} / \mathrm{min}$ at T0 to $28.79 \pm 6.59$ $\mathrm{mL} / \mathrm{kg} / \mathrm{min}$ at T1 $(P=0.06)$. Physical functioning $(P=0.002)$, physical limitation $(P=0.048)$, general health $(P<0.001)$ and bodily pain $(P=0.030)$ were improved at T1. A significant improvement occurred in dimensions of HP such as physical condition $(P=0.001)$, adiposity $(P<0.001)$, alimentation $(P<0.001)$, general health $(P=0.038)$, and perceived general health $(P=0.001)$. In addition, there was an improvement in items of PSP such as self-perceived coordination ( $P=0.022)$, endurance $(P=0.001)$ and activity $(P=0.001)$, global self-concept $(P=0.015)$, and appearance $(P=0.016)$. Physical but not mental HR-QOL domains, HP and PSP were associated with weight reduction.

Conclusion: While HIIT favors improved HR-QOL, HP status and PSP in adolescents with obesity, physical but not mental HR-QOL, HP and PSP were associated with weight reduction.

Key words: High-intensity interval training, Quality of life, Health perception, Adolescents, Obesity
Received July 7, 2020

Reviewed July 31,2020

Accepted September 10, 2020

*Corresponding author

Marwa Khammassi

(iD

https://orcid.org/0000-0002-5509-5997

Clermont Auvergne University, EA 3533, Laboratory of the Metabolic Adaptations to Exercise under Physiological and Pathological Conditions (AME2P), BP 80026, F-63171 Aubière Cedex, France Tel: +33-4-73-40-76-79

Fax: +33-4-73-40-76-79

E-mail:

khammassimarwa.issep@hotmail.com 


\section{INTRODUCTION}

Pediatric obesity, resulting mainly from a physically inactive lifestyle and inappropriate dietary habits, is becoming one of the major public health problems of the 21 th century. ${ }^{1,2}$ Early prevention strategies remain a high priority given the negative health consequences of being overweight/obese during adolescence but also later in life. ${ }^{3}$ Indeed, pre-pubertal children with obesity have a $20 \%$ to $50 \%$ higher risk of remaining obese once adult, which rises to between $50 \%$ and $70 \%$ in pubertal adolescents with obesity. ${ }^{4}$ This rising prevalence of obesity is associated with a concomitant increase of several medical complications such as functional impairments, metabolic disorders and altered psychological health among others, which has been well documented in the literature. ${ }^{5,6}$

The prevention and the treatment of obesity, which is considered a multifactorial disorder, requires effective multifaceted interventions. ${ }^{7}$ Multidisciplinary weight-loss interventions combining physical activity, nutritional guidelines and psychological support have been recommended and found effective for the treatment of pediatric obesity and its related complications (physical and psychological complications). ${ }^{8,9}$ However, evidence regarding the effect of such interventions on health-related quality of life (HRQOL) in children and adolescents remains scarce. Recently, Khammassi et al. ${ }^{10}$ found improvements in the main dimensions of HRQOL, such as physical functioning, general health, physical score and mental score, in adolescents with obesity who underwent a 10-month multidisciplinary weight loss program. In addition to quality of life, which basically refers to wellbeing, the authors also assessed the effects of their intervention on the adolescents' perception of their health, which could be defined as what they selfbelieve about their own physical fitness, weight, diet, sleep quality or level of stress. ${ }^{11,12}$ According to their results, the adolescents also improved their overall health perception (HP). Freitas et al. ${ }^{13}$ also found improved HR-QOL in adolescents with obesity in response to a 12 -week multidisciplinary weight management program combined with clinical counseling, physical activity (moderate aerobic training), and nutritional education with or without psychological support. Interestingly, these HR-QOL benefits were greater in the group of adolescents who received psychological counseling. ${ }^{13}$

While most of the available studies used multidisciplinary inter- ventions that included moderate-intensity aerobics plus resistance exercises, more recent studies compared the effect of different physical activity programs. For instance, Lopera et al. ${ }^{14}$ compared the effect of 16 weeks of land versus water-based physical interventions of similar work volume, in adolescents with obesity. While both interventions improved HR-QOL, the land-based program had particular effects on the social and psychosocial dimensions of HR-QOL as well as on the total HR-QOL score compared with the water-based program. Similarly, Rey et al. ${ }^{15}$ assessed the effect of 5 weeks vigorous interval training combined with dietary counseling on the physical self-perception (PSP) of adolescents with obesity demonstrating improved self-perception of endurance, activity level, sport competence, global physical self-concept and appearance.

Although a lot of attention has been given to the use of high-intensity interval training (HIIT) as an effective strategy among adolescents with obesity, ${ }^{16-18}$ to our knowledge, the psychological responses to this exercise modality in this population remains unknown. In 2017, Svensson et al. ${ }^{19}$ compared the effects of 16 weeks HIIT or moderate-intensity training on HR-QOL among adults with obesity. While both groups improved overall HR-QOL, greater effects were observed in the HIIT group, particularly regarding physical functioning, vitality and general health, improvements that were however not associated with changes in body mass of aerobic capacities.

Since the achievement and maintenance of weight loss may rely mainly on the psychological responses to our interventions, it is of high importance to improve the assessment and understanding of the responses to HIIT in adolescents with obesity. Therefore, the first aim of the present study was to examine for the first time the impact of a 16-week HIIT on HR-QOL, HP, and PSP in adolescents with obesity. The second aim was to explore whether observed changes were associated with changes in body weight, body composition and aerobic capacity.

\section{METHODS}

\section{Population}

Adolescents with obesity were recruited from an inpatient weight-management intervention (Pediatric Obesity Service Sesa- 
me, Centre Médical Infantile de Romagnat, Romagnat, France). Fifty-six adolescents ( 28 girls and 28 boys; mean body mass index [BMI], $35 \pm 4.89 \mathrm{~kg} / \mathrm{m}^{2}$; mean BMI z-score, $2.34 \pm 0.31$; BMI percentile, $98.58 \pm 0.95$ ) aged from 11-17 years were screened for the study. The main inclusion criteria were primary obesity (BMI $>$ 95th percentile of the Centers for Disease Control reference charts; Centers for Disease Control and Prevention 1999-2000); no pre-existing medical condition that would restrict participants from engaging in regular exercise; and not being engaged in more than 2 hours of structured physical activity per week (International Physical Activity Questionnaire).

All experiments were performed in accordance with the principles of the Declaration of Helsinki and were formally approved by the local Ethical Committee (CPP Sud Est VI: AU1178; Clinical Trial NCT02482220). Adolescents and their legal representatives were given study information sheets and provided dsigned informed consent.

\section{Study design}

All adolescents were medically screened by a pediatrician to ensure appropriate recruitment. Subsequently, their baseline anthropometric profile, body composition (measured by dual X-ray absorptiometry $[\mathrm{DXA}]$ ) and individual aerobic capacities (maximal oxygen consumption $\left[\mathrm{VO}_{2 \text { peak }}\right]$ ) were assessed. The HR-QOL (36item short form survey [SF-36]), HP (HP questionnaire) and PSP (PSP questionnaire) self-reported by the adolescents were also evaluated. The adolescents then joined the local Pediatric Obesity Center (Service Sesame, Centre Médical Infantile de Romagnat, Romagnat, France) for a 4-month inpatient multidisciplinary weight-management program combining nutritional counseling, physical training (HIIT exercises+leisure-time activities), and health-related therapeutic education. All measurements were replicated by the end of the 16-week intervention.

\section{Anthropometric measurements and body composition}

Body weight and height were measured according to the recommended procedure in indoor clothing and without shoes, and they were recorded to the nearest $0.1 \mathrm{~kg}$ and $0.5 \mathrm{~cm}$, respectively. Subjects were weighed on a reliable digital scale (SECA, Les Mureaux, France). Height was measured by a standard wall-mounted stadi- ometer (SECA). BMI was calculated as body weight $(\mathrm{kg})$ divided by height $(\mathrm{m})$ squared $\left(\mathrm{kg} / \mathrm{m}^{2}\right)$; and reported on appropriate growth curves. Body composition was obtained using DXA following standardized procedures (QDR4500A scanner; Hologic, Waltham, MA, USA).

\section{Maximal oxygen uptake test $\left(\mathrm{VO}_{2}\right)$}

Each participant performed an incremental exercise test to exhaustion on a traditional concentric ergometer ${ }^{20}$ to determine $\mathrm{VO}_{\text {2peak }}$. The test consisted of pedaling for 3 minutes with an initial power of $30 \mathrm{~W}$, followed by increments in power of $15 \mathrm{~W}$ at 1-minute intervals until exhaustion. Participants were requested to wear a polar heart rate monitor (Polar V800) during the test to observe maximal heart rate percentage throughout the test. Cardiac electrical activity (Ultima Series, Saint Paul, MN, USA) was monitored in addition to breath by breath gas exchange (BreezeSuite, Saint Paul, MN, USA) to determine $\mathrm{VO}_{2}$ and carbon dioxide $\left(\mathrm{CO}_{2}\right)$ production $\left(\mathrm{VCO}_{2}\right)$. Volumes and gases were calibrated before each test. The validation of $\mathrm{VO}_{2 \text { peak }}$ attainment was based on these criteria: (1) heart rate $>90 \%$ of the theoretical maximum heart rate (210-0.65 $\times$ age), (2) respiratory exchange ratio $\left(\mathrm{VCO}_{2} / \mathrm{VO}_{2}\right)>1.1$ and $/$ or $\mathrm{VO}_{2}$ plateau despite increasing exercise intensity. When the subject could no longer maintain the required speed imposed, the test was stopped and the $\mathrm{VO}_{2}$ peak was defined as the average of the last 30 seconds of exercise before exhaustion.

\section{Multidisciplinary weight loss program}

The 16-week inpatient multidisciplinary weight-loss intervention combined physical exercise (4/wk), nutritional education $(2 / \mathrm{mo})$ and psychological support $(1 / \mathrm{mo})$. The adolescents received an isocaloric diet based on recommendations for their age and sex. ${ }^{21}$ Physical activity included aquatic activities $(1 / \mathrm{wk})$, leisure-time activities (e.g., ball games; $1 / \mathrm{wk}$ ) and HIIT+strength training (2/wk). In addition, the adolescents performed 2 hours of physical education per week at school. The HIIT session started with 15 minutes intermittent exercise on an ergometer bicycle, alternating 30 seconds intense exercise and 30 seconds active recovery (free but compulsory pedaling), also followed by strength training ( 2 times/wk). After a 5-minute warm-up set at $60 \%$ of the adolescents $\mathrm{VO}_{2 \text { peak, }}$, the intensity of the HIIT exercise followed a progressive increase 
from $75 \%$ of baseline $\mathrm{VO}_{2 \text { peak, }}$ ending at $90 \%$. The cycling intensity of HIIT was controlled using mechanical workload settings. Regarding the strength training, tests were realized for each of the selected exercises to determine the participants maximal 10 repetitions. The exercises were the bench press, pulley (lat pull-down), leg-press, curl, ankle extension machine (sitting), curl machine for triceps, abdominal machine and trunk extensor machine. The adolescents had to perform 10 repetitions of each exercise interspersed by one minute of rest; and to repeat this three times, with a rest period of 4 minutes between each round. The training intensity was set individually and progressively increased from $65 \%$ to $85 \%$ of 10 maximal repetitions at the post-intervention. This training program has already been implemented with success in this population. $^{22}$

\section{Health-related quality of life}

The SF-36 that was developed by Ware and Sherbourne was used in our study to assess HR-QOL in adolescents. The SF-36 evaluates health based on scores of $0-100$ with higher scores indicating a good state of QOL. Based on 36 items, this questionnaire measures eight domains: general health, physical functioning, physical role, pain, vitality, social functioning, role emotional, and mental health. ${ }^{23}$ A physical component scale (PCS) and mental component scale (MCS) can then be calculated..$^{24}$ The validity of this questionnaire has been previously reported in adolescents with obesity. ${ }^{10}$

\section{Health perception (health perception scale)}

All adolescents were asked to complete a short self-administered questionnaire specifically designed to explore their perception of health ("HP scale"). Six criteria were investigated: perceived (1) physical fitness, (2) ideal weight, (3) healthy balanced diet, (4) sleep quality, (5) stress level, and (6) general health. A 10-point scale from 1 (not at all) to 10 (very much) was used to assess each item. The six individual scores were computed to obtain a global score for HP. This questionnaire has been shown to provide satisfactory results in both adults ${ }^{11,21}$ and adolescents with obesity. ${ }^{10}$

\section{Physical self-perception}

PSPs were measured with the French version of the short form of the physical self-description questionnaire. ${ }^{25}$ This questionnaire contains 40 items that measure 11 physical self-dimensions: coordination, strength, flexibility, endurance, global self-esteem, health, activity, body fat, sport competence, global physical self-concept, and appearance. A 6-point scale from 1 (false) to 6 (true) was used to assess each item. The eleven individual scores were computed to obtain a global score for PSP. The validity of this questionnaire in children and adolescents with obesity has been reported previously. ${ }^{15}$

\section{Statistical procedures}

The sample size estimation was determined according to (1) the CONSORT 2010 statement and (2) Cohen's recommendations, ${ }^{26}$ who has defined effect-size (ES) bounds as small (ES 0.2), medium (ES 0.5) and large (ES 0.8, "grossly perceptible and therefore large"). Therefore, with more than 50 participants at each time point evaluation (baseline and at the post-intervention), an ES greater than 0.5 can be hypothesized for a two-tailed type I error at $5 \%$, a statistical power greater than $95 \%$ and an intra-individual correlation coefficient at 0.5 to take into account between and within participant variability.

Statistical analyses were performed using Stata version 15 (StataCorp., College Station, TX, USA). All tests were two-tailed, with a type I error set at 0.05 . Continuous data were expressed as mean \pm standard deviation. The assumption of normality was assessed by using the Shapiro-Wilk test. The comparisons between baseline and post-intervention were performed using Student paired $t$-test or Wilcoxon's test when the assumptions of the t-test were not met. As proposed by $\mathrm{Feise}^{27}$ a particular focus has been also given to the magnitude of differences, in addition to inferential statistical tests expressed using $P$-values and Hedges' ESs with 95\% confidence intervals. Typical error and intra-class coefficients (ICCs) for the differences between baseline and $\mathrm{T} 1$ are also provided. Then, the changes between baseline and the post-intervention were calculated. The study of relationships between anthropometric, body composition and aerobic capacity variations and HR-QOL, HP, and PSP changes was conducted using Pearson or Spearman coefficient correlation, applying a Sidak's type I error correction to take into account multiple comparisons. 


\section{RESULTS}

\section{Anthropometric measurements, body composition, and} aerobic capacities

As detailed in Table 1, body weight, BMI and fat mass percentage were all significantly reduced by the intervention $(P<0.001)$, while fat-free mass (FFM, $\mathrm{kg}$ ) and $\mathrm{VO}_{2 \text { peak }}$ remains unchanged $(P=0.083$ and $P=0.062$, respectively).

\section{Health perception}

Data related to changes in HP during the 16-week intervention are summarized in Table 2. Significant improvements were observed in the dimensions of HP, such as physical condition $(P<0.001)$, adiposity $(P<0.001)$, alimentation $(P<0.001)$, general health $(P=$ $0.038)$, and general health perceived $(P=0.001)$. Neither sleep nor stress were improved by the intervention.

\section{Health-related quality of life}

Data related to changes in HR-QOL during the 16-week intervention are presented in Table 3. Significant improvements were observed in the dimensions of HR-QOL after the program, such as physical functioning $(P=0.002)$, physical limitations $(P=0.048)$, bodily pain $(P=0.030)$, general health $(P<0.001)$, and PCS $(P<$ $0.001)$. None of the other dimensions of the SF-36 questionnaire were significantly modified.

\section{Physical self-perception}

Table 4 details the effect of the intervention on the PSP components. Self-perceived coordination $(P=0.022)$, endurance $(P=$ $0.001)$, activity $(P<0.001)$, global self-concept $(P=0.015)$, and appearance $(P=0.016)$ were significantly improved while perceived sport competence and perceived body fat almost reached significance ( $P=0.054$ and $P=0.064$ respectively). Whereas the other dimensions of the questionnaire did not significantly change, the global computed PSP score was significantly improved $(P=0.006)$.

Correlations between anthropometric, body composition and aerobic capacities variations with HR-QOL, HP, and PSP changes

The heatmap in Fig.1 presents the different correlations obtained

Table 1. Effects of the HIIT program on anthropometric measurements, body composition and aerobic capacity in obese adolescents

\begin{tabular}{lcccccc}
\hline Variable & T0 & T1 & $P$ & TE & ICC & Hedges's \\
\hline Weight $(\mathrm{kg})$ & $92.69 \pm 18.90$ & $85.97 \pm 16.27$ & $<0.001$ & 0.03 & 0.97 & $-1.52(-1.81$ to-1.23) \\
BMI (kg/m²) & $35 \pm 4.89$ & $32.11 \pm 4.50$ & $<0.001$ & 0.03 & 0.95 & $-1.92(-2.22$ to -1.63$)$ \\
BMl percentile & $98.58 \pm 0.95$ & $97.61 \pm 2.39$ & $<0.001$ & 0.01 & 0.45 & $-0.54(-0.83$ to -0.25$)$ \\
Z score & $2.34 \pm 0.31$ & $2.11 \pm 0.36$ & $<0.001$ & 0.06 & 0.87 & $-1.16(-1.41$ to -0.90$)$ \\
FM (\%) & $36.05 \pm 9.15$ & $30.40 \pm 7.86$ & $<0.001$ & 0.05 & 0.96 & $-1.35(-1.64$ to -1.06$)$ \\
FFM (kg) & $54.99 \pm 11.47$ & $53.97 \pm 10.43$ & 0.083 & 0.04 & 0.96 & $-0.26(-0.55$ to 0.03$)$ \\
VO $_{2 \text { peak }}(\mathrm{mL} / \mathrm{kg} / \mathrm{min})$ & $26.35 \pm 5.81$ & $28.79 \pm 6.59$ & 0.062 & 0.24 & 0.95 & $0.29(-0.01$ to 0.60$)$ \\
\hline
\end{tabular}

Values are presented as mean \pm standard deviation.

HIIT, high-intensity interval training; TO, baseline; T1, post-intervention; TE, typical error; ICC, intra-class coefficient; BMI, body mass index; FM, fat mass; FFM, fat-free mass; VO2peak, maximal oxygen consumption.

Table 2. Effects of HIIT program on health perception in obese adolescents

\begin{tabular}{lcccccc}
\hline Variable & T0 & T1 & $P$ & TE & ICC & Hedges's \\
\hline Physical condition & $4.96 \pm 2.69$ & $6.39 \pm 2.05$ & $<0.001$ & 1.46 & 0.33 & $0.53(0.23$ to 0.82$)$ \\
Adiposity & $2.47 \pm 1.89$ & $4.14 \pm 1.88$ & $<0.001$ & 1.05 & 0.50 & $0.91(0.61$ to 1.21$)$ \\
Alimentation & $4.63 \pm 2.30$ & $6.25 \pm 2.27$ & $<0.001$ & 1.47 & 0.33 & $0.57(0.35$ to 0.94$)$ \\
Sleep & $6.65 \pm 3.18$ & $6.18 \pm 2.82$ & 0.230 & 0.89 & 0.36 & $-0.18(-0.48$ to 0.11$)$ \\
Stress & $5.96 \pm 3.03$ & $6.30 \pm 3.18$ & 0.529 & 1.21 & 0.28 & $0.09(-0.20$ to 0.39$)$ \\
General health & $6.04 \pm 2.57$ & $6.89 \pm 2.10$ & 0.038 & 1.17 & 0.30 & $0.31(0.02$ to 0.61$)$ \\
General health perceived & $5.13 \pm 1.43$ & $6.02 \pm 1.42$ & 0.001 & 0.59 & 0.20 & $0.52(0.22$ to 0.81$)$ \\
\hline
\end{tabular}

Values are presented as mean \pm standard deviation.

HIIT, high-intensity interval training; TO, baseline; T1, post-intervention; TE, typical error; ICC, intra-class coefficient. 
Table 3. Effects of HIIT program on health-related quality of life in obese adolescents

\begin{tabular}{lcccccc}
\hline Variable & T0 & T1 & $P$ & TE & ICC & Hedges's \\
\hline Physical functioning & $75.10 \pm 24.72$ & $83.84 \pm 20.26$ & 0.002 & 0.26 & 0.73 & 0.48 (0.18 to 0.78$)$ \\
Physical limitation & $51.92 \pm 25.17$ & $59.88 \pm 28.94$ & 0.048 & 0.57 & 0.36 & 0.30 (0.00 to 0.60$)$ \\
Bodily pain & $76.49 \pm 23.50$ & $85.35 \pm 18.32$ & 0.030 & 0.39 & 0.23 & 0.33 (0.03 to 0.63$)$ \\
General health & $57.17 \pm 16.69$ & $68.31 \pm 16.55$ & $<0.001$ & 0.22 & 0.57 & $0.73(0.43$ to 1.03$)$ \\
PCS score & $65.30 \pm 13.70$ & $74.35 \pm 14.07$ & $<0.001$ & 0.22 & 0.55 & $0.71(0.41$ to 1.01$)$ \\
Social functioning & $70.83 \pm 21.16$ & $75.29 \pm 24.01$ & 0.321 & 0.47 & 0.13 & $0.15(-0.15$ to 0.45$)$ \\
Emotional limitation & $57.14 \pm 35.36$ & $58.73 \pm 38.82$ & 0.990 & 0.54 & 0.30 & $0.00(-0.30$ to 0.30$)$ \\
Vitality & $57.90 \pm 15.05$ & $58.37 \pm 16.10$ & 0.838 & 0.49 & 0.41 & $0.03(-0.27$ to 0.33$)$ \\
Mental health & $56.57 \pm 16.84$ & $58.05 \pm 16.41$ & 0.764 & 0.25 & 0.43 & $0.05(-0.25$ to 0.35$)$ \\
MCS core & $60.45 \pm 16.91$ & $62.52 \pm 17.44$ & 0.894 & 0.31 & 0.55 & $0.04(-0.28$ to 0.33$)$ \\
\hline
\end{tabular}

Values are presented as mean \pm standard deviation.

HIIT, high-intensity interval training; T0, baseline; T1, post-intervention; TE, typical error; ICC, intra-class coefficient; PCS, physical component scale; MCS, mental component scale.

Table 4. Effects of HIIT program on physical self-perception in obese adolescents

\begin{tabular}{lcccccc}
\hline Variable & T0 & T1 & $P$ & TE & ICC & Hedges's \\
\hline Coordination & $3.26 \pm 1.29$ & $3.94 \pm 1.22$ & 0.022 & 0.75 & 0.20 & $0.43(0.07$ to 0.80$)$ \\
Strength & $3.69 \pm 1.35$ & $4.18 \pm 1.19$ & 0.096 & 0.55 & 0.17 & $0.31(-0.06$ to 0.68$)$ \\
Flexibility & $3.00 \pm 1.42$ & $3.21 \pm 1.42$ & 0.568 & 0.51 & 0.60 & $0.11(-0.27$ to 0.49$)$ \\
Endurance & $2.53 \pm 1.32$ & $3.54 \pm 1.33$ & 0.001 & 1.06 & 0.20 & $0.65(0.28$ to 1.02$)$ \\
Global self-esteem & $3.44 \pm 0.80$ & $3.67 \pm 0.65$ & 0.173 & 0.26 & 0.25 & $0.26(-0.12$ to 0.62$)$ \\
Health & $2.53 \pm 1.16$ & $1.93 \pm 1.37$ & 0.239 & 0.09 & 0.97 & $0.22(-0.16$ to 0.60$)$ \\
Activity & $2.62 \pm 1.64$ & $3.59 \pm 1.62$ & $<0.001$ & 0.92 & 0.61 & $0.70(0.34$ to 1.05$)$ \\
Body fat & $5.13 \pm 1.18$ & $4.50 \pm 1.52$ & 0.064 & 0.44 & 0.44 & $-0.34(-0.71$ to 0.02$)$ \\
Sport competence & $3.31 \pm 1.76$ & $4.00 \pm 1.53$ & 0.054 & 0.85 & 0.21 & $0.35(0.00$ to 0.70$)$ \\
Global self-concept & $2.78 \pm 1.54$ & $3.55 \pm 1.67$ & 0.015 & 0.87 & 0.48 & $0.44(0.09$ to 0.80$)$ \\
Appearance & $2.83 \pm 1.30$ & $3.61 \pm 1.71$ & 0.016 & 0.43 & 0.55 & $0.45(0.08$ to 0.82$)$ \\
PSP general score & $3.20 \pm 0.83$ & $3.72 \pm 0.80$ & 0.006 & 0.20 & 0.57 & $0.67(0.28$ to 1.07$)$ \\
\hline
\end{tabular}

Values are presented as mean \pm standard deviation.

HIIT, high-intensity interval training; TO, baseline; T1, post-intervention; TE, typical error; ICC, intra-class coefficient; PSP, physical self-perception.

between the variables in the study. Regarding HP, negative correlations between $\Delta$ weight and $\Delta$ physical condition $(\mathrm{r}=-0.33, P<$ $0.05), \Delta$ general health $(\mathrm{r}=-0.39, P<0.05)$, and $\Delta$ general health perceived $(\mathrm{r}=-0.42, P<0.05)$ were observed. Similarly, negative relationships were found between $\triangle \mathrm{BMI}$ and $\Delta$ physical conditions $(\mathrm{r}=-0.46, P<0.05), \Delta$ alimentation $(\mathrm{r}=-0.37, P<0.05)$, and $\Delta$ general health perceived $(\mathrm{r}=-0.35, P<0.05)$. The $\Delta$ FFM was negatively correlated with $\Delta$ adiposity $(\mathrm{r}=-0.33, P<0.05)$ and $\Delta \mathrm{VO}_{2 \text { peak }}$ was correlated negatively with $\Delta$ physical condition $(\mathrm{r}=-0.40, P<0.05)$ and $\Delta$ stress $(\mathrm{r}=-0.39, P<0.05)$.

Regarding HR-QOL, $\triangle$ weight was found to correlate negatively with $\Delta$ general heath $(\mathrm{r}=-0.45, P<0.05)$, and $\Delta$ vitality $(\mathrm{r}=-0.37$, $P<0.05)$. Similarly, a negative correlation was observed between $\triangle \mathrm{BMI}$ and $\Delta$ physical functioning $(\mathrm{r}=-0.38, P<0.05), \Delta$ general health $(\mathrm{r}=-0.49, P<0.05), \Delta$ physical score $(\mathrm{r}=-0.34, P<0.05)$, and $\Delta$ vitality $(\mathrm{r}=-0.36, P<0.05)$. $\Delta$ Zscore was correlated negatively with physical functioning $(\mathrm{r}=-0.34, P<0.05)$. Finally, regarding $\Delta$ weight, the $\Delta \mathrm{Zscore}, \Delta \mathrm{BMI}$ percentile and $\Delta \mathrm{VO}_{2 \text { peak }}$ were positively correlated with $\Delta$ body fat $(r=0.44, r=0.59, r=0.49$, and $\mathrm{r}=0.53$, respectively, $P<0.05)$ and $\triangle \mathrm{FFM}$ was negatively correlated with $\Delta$ flexibility $(\mathrm{r}=-0.41, P<0.05)$.

\section{DISCUSSION}

The main goal of the present study was to question the effects of 16 weeks of HIIT as a component of a multidisciplinary weight-loss intervention, on HR-QOL, HP, and PSP in adolescents with obesity. According to our results, the intervention favored improvements in 

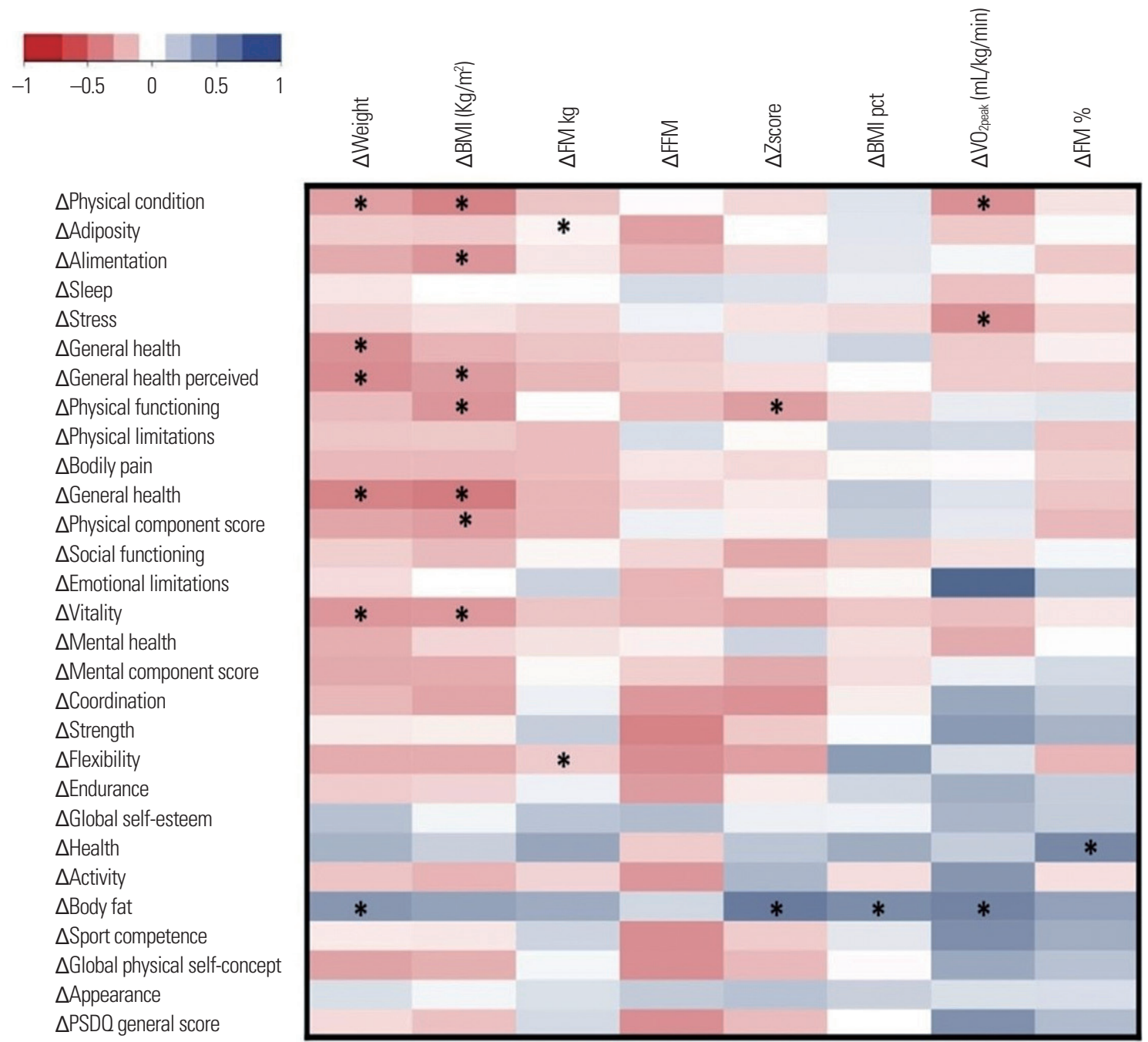

Figure 1. Heatmap representation of the correlations between changes $(\Delta)$ of the items and scores for health-related quality of life, health perception, physical self-perception, anthropometrics, body composition variables, and maximal aerobic capacities $\left(\mathrm{VO}_{2 \text { peak }}\right.$, between baseline (T0) and post-intervention (T1). The darkest is the box and the higher is the correlation. ${ }^{*} P<0.05$. BMl, body mass index; FM, fat mass (\% and kg); FFM, fat-free mass (kg); $B M l$ pct, BMl percentile; $V_{\text {peak, }}$ maximal oxygen consumption; PSDQ, physical self-perception description questionnaire.

the physical dimension of quality of life as well as in the HP and PSP of the adolescents. In addition, our results suggest that the improvements in the physical dimension of HR-QOL, HP, and PSP, might be associated with the observed changes in body weight. The adolescents showed marked improvement in body composition and HP, which may, in turn, have led to improvements in perceived physical condition, adiposity, alimentation, general health and perceived general health. The physical functioning, physical limitations, bodily pain, and general health items of the SF-36 and PCS increased significantly with the intervention, while the mental dimension of the SF-36 questionnaire remained unchanged. In addition, our HIIT intervention was effective in improving some dimensions of the PSP questionnaire, such as coordination, endurance, activity, sport competence, global physical self-concept, appearance and the PSP general score.

While the effect of weight-loss interventions on body composition, metabolic profile, or physical fitness have been widely questioned among adolescents with obesity, their psychological and mental health impact remain poorly explored. HR-QOL is reported to be inversely associated with weight gain and the risk of obesity in pediatric populations ${ }^{28}$ and there is a strong association between the degree of obesity and quality of life among children and adolescents. ${ }^{29}$ Previous studies have demonstrated that a reduction in weight is associated with an improvement in HR-QOL, ${ }^{30,31}$ and 
improvements in some aspects of HR-QOL have been observed and associated with a weight loss above $5 \% .{ }^{32}$ While some studies have shown an improvement in the physical and mental dimensions of the SF-36 after weight-loss interventions in adolescents with obesity, ${ }^{10,33}$ others have only observed an improvement in the physical dimensions. ${ }^{12,34}$ The present work is in line with these latter studies and seems to be the first one to question the effect of HIIT on HR-QOL in adolescents with obesity. As previously underlined in adults, ${ }^{19}$ our results demonstrated that 16 weeks of HIIT induces a significant improvement in the physical dimension of HR-QOL only, resulting in the improvement of the physical functioning, physical limitations, bodily pain, and general health items of the SF-36 and of its global PCS.

In addition, the results of the present study have demonstrated an improvement in HP in adolescents who suffer from obesity; HP refers to the perception that individuals have of their own overall health. ${ }^{35}$ This greater HP by the post-intervention results from the improvements of the adolescents' perceived physical condition, adiposity, alimentation, general health, and perceived general health. Our results are in line with the extant literature, even though the available evidence remains limited. Khammassi et al. ${ }^{10}$ showed a significant improvement in perceived physical condition, adiposity, healthy balanced diet, general health, and the general HP score after 5 and 10 months of a multidisciplinary weight-loss intervention among adolescents with obesity.

In our sample, HIIT not only seemed to improve HR-QOL and $\mathrm{HP}$, it also induced positive effects on the PSP of the adolescents. In 2017, Rey et al. ${ }^{15}$ showed that a 5-week intervention combining vigorous interval training with a dietary education led to a better PSP in adolescent boys and girls with obesity, particularly in their perception of their endurance, physical activity, sport competence, self-concept, and appearance. Interestingly, this improved perception of their physical abilities was not concordant with the objectively assessed physical fitness of the adolescents. Indeed, the perceived fitness of the adolescent girls increased in response to the intervention, despite unchanged performances in their fitness tests. ${ }^{15}$ Although Rey et al. ${ }^{15}$ used a combination of various physical exercise performed at high intensity, they did not implement a proper HIIT and the present results are the first to question PSP in response to this modality in adolescents with obesity. In line with
Rey et al., ${ }^{15}$ our results suggest that 16 weeks of HIIT have positive effects on overall PSP in this population. Interestingly, better scores were observed regarding the physical subcomponents of the PSP while the perception of global self-esteem, global health and body fat percentage remained unchanged.

In addition to the observed beneficial effects of our HIIT intervention on overall HR-QOL, HP and PSP; body weight, BMI, and body fat percentage were improved while FFM and $\mathrm{VO}_{2 \text { peak }}$ did not change. The positive effect on body composition and body weight is in line with previously published studies and meta-analyses. ${ }^{3,16}$ However, the absence of improvement in FFM and $\mathrm{VO}_{2 \text { peak }}$ seems in contradiction with other studies. ${ }^{36,37}$ Importantly, our results did not reach significance, both FFM and $\mathrm{VO}_{2 \text { peak }}$ showed a tendency that might be due to the inter-individual variability of our sample. Our results, however, seem to indicate that the observed improvement in HR-QOL, HP, and PSP might not be associated with the observed changes in body weight, body composition, and physical fitness. While the changes in most of the HR-QOL components showed some associations with body weight loss and BMI decrease in the adolescents, no association was found for the changes in

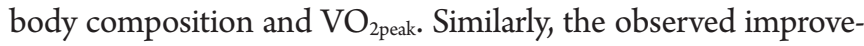
ments of HP and PSP were not associated with anthropometric and body composition changes or with the variation in $\mathrm{VO}_{2 \text { peak }}$. This corresponds with recent results from our team suggesting that the HR-QOL and HP response to a weight-loss intervention including physical activity might only be slightly related to body weight, fat mass and FFM changes. ${ }^{10}$ This seems to agree with the results proposed by Rey et al. ${ }^{15}$ who suggest that the psychological perception and health of the adolescents might be improved in response to weight loss, without concomitant improvements in objectively measured physical characteristics or performances.

Although the present study is the first to report evidence regarding the psycho-physiological responses to HIIT in adolescents with obesity, these results should be considered in light of some limitations. First, the clinical nature of the present results did not allow the inclusion of a control group following another type of exercise training (such as moderate-intensity continuous exercise, for instance) or of a group of adolescents without an intervention. Second, while heart rate was continuously measured during the session as a double method to control for the exercise intensity (in ad- 
dition to the mechanical load imposed on the ergocycle) it was not recorded, which could have provided important additional supporting information. Another limitation is the lack of evaluation of the muscle strength and fitness of adolescents. Indeed, while we assessed $\mathrm{VO}_{2 \text { peak }}$, it would have been interesting to also question whether the observed psychological improvements could be associated with better muscle capacities. As part of their clinical intervention, the adolescents received a normocaloric diet respecting the recommendation for their age while it would have been better to individualize their diet based on energetic needs, which was not possible for practical reasons. Although the inpatient nature of the intervention composes a methodological strength since it allows a high level of control on the compliance of the adolescents to the program and of their daily activities outside the program; it also restricts the interpretation and applicability of the results to other contexts such as the adolescents' daily home-based free-living environment, or to the school setting where adolescents usually spend most of their time.

The present study is the first to evaluate the impact of HIIT as a component of a multidisciplinary weight-loss intervention on HRQOL, HP, and PSP in adolescents with obesity. According to our results, a 4-month HIIT intervention favors HR-QL, HP, and PSP improvements. Physical HR-QOL but not mental HR-QOL domains, HP, and PSP were associated with weight reduction; none of them were associated with changes in aerobic fitness. Further studies are necessary to better understand the effect of physical activity interventions and particularly HIIT on these psycho-physiological variables in adolescents with obesity.

\section{CONFLICTS OF INTEREST}

The authors declare no conflict of interest.

\section{AUTHOR CONTRIBUTIONS}

Study concept and design: DT; acquisition of data: all authors; analysis and interpretation of data: all authors; drafting of the manuscript: MK; critical revision of the manuscript: all authors; statistical analysis: BP; administrative, technical, or material support: all authors; and study supervision: DT.

\section{REFERENCES}

1. Farpour-Lambert NJ, Baker JL, Hassapidou M, Holm JC, Nowicka P, O'Malley G, et al. Childhood obesity is a chronic disease demanding specific health care: a position statement from the Childhood Obesity Task Force (COTF) of the European Association for the Study of Obesity (EASO). Obes Facts 2015;8:342-9.

2. Styne DM. Childhood and adolescent obesity: prevalence and significance. Pediatr Clin North Am 2001;48:823-54.

3. Hoare E, Fuller-Tyszkiewicz M, Skouteris H, Millar L, Nichols M, Allender S. Systematic review of mental health and well-being outcomes following community-based obesity prevention interventions among adolescents. BMJ Open 2015;5: e006586.

4. Tounian P, Girardet JP. Obesity in children: a disease that risks the vital prognosis. Arch Pediatr 2001;8:7-10.

5. Rendón-Macías ME, Rosas-Vargas H, Villasís-Keever MÁ, Pérez-García C. Children's perception on obesity and quality of life: a Mexican survey. BMC Pediatr 2014;14:131.

6. Hruby A, Manson JE, Qi L, Malik VS, Rimm EB, Sun Q et al. Determinants and consequences of obesity. Am J Public Health 2016;106:1656-62.

7. Zhang ZY, Wang MW. Obesity, a health burden of a global nature. Acta Pharmacol Sin 2012;33:145-7.

8. Seo YG, Lim H, Kim Y, Ju YS, Lee HJ, Jang HB, et al. The Effect of a multidisciplinary lifestyle intervention on obesity status, body composition, physical fitness, and cardiometabolic risk markers in children and adolescents with obesity. Nutrients 2019;11:137.

9. Rank M, Wilks DC, Foley L, Jiang Y, Langhof H, Siegrist M, et al. Health-related quality of life and physical activity in children and adolescents 2 years after an inpatient weight-loss program. J Pediatr 2014;165:732-7.e2.

10. Khammassi M, Miguet M, O’Malley G, Fillon A, Masurier J, Damaso AR, et al. Health-related quality of life and perceived health status of adolescents with obesity are improved by a 10-month multidisciplinary intervention. Physiol Behav 2019; 210:112549.

11. Garnier S, Gaubert I, Joffroy S, Auneau G, Mauriège P. Im- 
pact of brisk walking on perceived health evaluated by a novel short questionnaire in sedentary and moderately obese postmenopausal women. Menopause 2013;20:804-12.

12. Hunt SM, McKenna SP, McEwen J, Backett EM, Williams J, Papp E. A quantitative approach to perceived health status: a validation study. J Epidemiol Community Health 1980;34: 281-6.

13. Freitas CR, Gunnarsdottir T, Fidelix YL, Tenório TR, Lofrano-Prado MC, Hill JO, et al. Effects of a psychological intervention on the quality of life of obese adolescents under a multidisciplinary treatment. J Pediatr (Rio J) 2017;93:185-91.

14. Lopera CA, da Silva DF, Bianchini JA, Locateli JC, Moreira AC, Dada RP, et al. Effect of water- versus land-based exercise training as a component of a multidisciplinary intervention program for overweight and obese adolescents. Physiol Behav 2016;165:365-73.

15. Rey O, Vallier JM, Nicol C, Mercier CS, Maïano C. Effects of combined vigorous interval training program and diet on body composition, physical fitness, and physical self-perceptions among obese adolescent boys and girls. Pediatr Exerc Sci 2017;29:73-83.

16. Thivel D, Masurier J, Baquet G, Timmons BW, Pereira B, Berthoin S, et al. High-intensity interval training in overweight and obese children and adolescents: systematic review and meta-analysis. J Sports Med Phys Fitness 2019;59:310-24.

17. Gutin B, Yin Z, Humphries MC, Barbeau P. Relations of moderate and vigorous physical activity to fitness and fatness in adolescents. Am J Clin Nutr 2005;81:746-50.

18. Gibala MJ, McGee SL. Metabolic adaptations to short-term high-intensity interval training: a little pain for a lot of gain? Exerc Sport Sci Rev 2008;36:58-63.

19. Svensson S, Eek F, Christiansen L, Leif Wisén A. The effect of different exercise intensities on health related quality of life in people classified as obese. Eur J Physiother 2017;19:104-15.

20. Carnier J, de Mello MT, Ackel-DElia C, Corgosinho FC, Campos RM, Sanches Pde L, et al. Aerobic training (AT) is more effective than aerobic plus resistance training $(\mathrm{AT}+\mathrm{RT})$ to improve anorexigenic/orexigenic factors in obese adolescents. Appetite 2013;69:168-73.

21. Genin PM, Degoutte F, Finaud J, Pereira B, Thivel D, Duclos
M. Effect of a 5-month worksite physical activity program on tertiary employees overall health and fitness. J Occup Environ Med 2017;59:e3-10.

22. Dâmaso AR, da Silveira Campos RM, Caranti DA, de Piano A, Fisberg M, Foschini D, et al. Aerobic plus resistance training was more effective in improving the visceral adiposity, metabolic profile and inflammatory markers than aerobic training in obese adolescents. J Sports Sci 2014;32:1435-45.

23. Ware JE Jr. SF-36 health survey update. Spine (Phila Pa 1976) 2000;25:3130-9.

24. Taft C, Karlsson J, Sullivan M. Do SF-36 summary component scores accurately summarize subscale scores? Qual Life Res 2001;10:395-404.

25. Maïano C, Morin AJ, Mascret N. Psychometric properties of the short form of the Physical Self-Description Questionnaire in a French adolescent sample. Body Image 2015;12:89-97.

26. Cohen J. Statistical power analysis for the behavioral sciences. 2nd ed. Hillsdale (NJ): Lawrence Erlbaum Associates; 1988.

27. Feise RJ. Do multiple outcome measures require p-value adjustment? BMC Med Res Methodol 2002;2:8.

28. McGavock JM, Torrance BD, McGuire KA, Wozny PD, Lewanczuk RZ. Cardiorespiratory fitness and the risk of overweight in youth: the Healthy Hearts Longitudinal Study of Cardiometabolic Health. Obesity (Silver Spring) 2009;17:1802-7.

29. Whitaker BN, Fisher PL, Jambhekar S, Com G, Razzaq S, Thompson JE, et al. Impact of degree of obesity on sleep, quality of life, and depression in youth. J Pediatr Health Care 2018; 32:e37-44.

30. Rippe JM, Price JM, Hess SA, Kline G, DeMers KA, Damitz S, et al. Improved psychological well-being, quality of life, and health practices in moderately overweight women participating in a 12-week structured weight loss program. Obes Res 1998;6:208-18.

31. Fontaine KR, Barofsky I, Andersen RE, Bartlett SJ, Wiersema L, Cheskin LJ, et al. Impact of weight loss on health-related quality of life. Qual Life Res 1999;8:275-7.

32. Samsa GP, Kolotkin RL, Williams GR, Nguyen MH, Mendel $\mathrm{CM}$. Effect of moderate weight loss on health-related quality of life: an analysis of combined data from 4 randomized trials of sibutramine vs placebo. Am J Manag Care 2001;7:875-83. 
33. Blissmer B, Riebe D, Dye G, Ruggiero L, Greene G, Caldwell M. Health-related quality of life following a clinical weight loss intervention among overweight and obese adults: intervention and 24 month follow-up effects. Health Qual Life Outcomes 2006;4:43.

34. Masurier J, Miguet M, Courteix D, Boirie Y, Duclos M, Thivel D. Effect of a 10-month multidisciplinary weight loss program on quality of life and health perception in adolescents with obesity. Proceedings of the European Congress on Obesity; 2017 May; Porto, Portugal.
35. Kaplan GA, Camacho T. Perceived health and mortality: a nine-year follow-up of the human population laboratory cohort. Am J Epidemiol 1983;117:292-304.

36. Koubaa A. Effect of intermittent and continuous training on body composition cardiorespiratory fitness and lipid profile in obese adolescents. IOSR J Pharm 2013;3:31-7.

37. Ingul CB, Tjonna AE, Stolen TO, Stoylen A, Wisloff U. Impaired cardiac function among obese adolescents: effect of aerobic interval training. Arch Pediatr Adolesc Med 2010;164: $852-9$. 